\title{
$\begin{array}{ll}\text { Research Square } & \text { Preprints are preliminary reports that have not undergone peer review. } \\ \text { They should not be considered conclusive, used to inform clinical practice, }\end{array}$ or referenced by the media as validated information.
}

\section{Risk Factors for Postpartum Hemorrhage After Elective Cesarean Delivery for Twin Pregnancy}

\section{xiao jie wan ( $\nabla 518006 @ z j u . e d u . c n)$}

Wome's Hospital,Zhejiang University School of Medicine https://orcid.org/0000-0001-6351-3055

Wei Zhao

Zhejiang University School of Medicine Women's Hospital

\section{Li Zhao}

Zhejiang University School of Medicine Women's Hospital

Hong Wen

Zhejiang University School of Medicine Women's Hospital

\section{Research article}

Keywords: twin pregnancy, elective cesarean delivery, postpartum hemorrhage, risk factors

Posted Date: February 13th, 2021

DOI: https://doi.org/10.21203/rs.3.rs-228054/v1

License: (c) (1) This work is licensed under a Creative Commons Attribution 4.0 International License. Read Full License 


\section{Abstract}

Objective: To identify the high risk factors associated with postpartum hemorrhage (PPH) after selective cesarean delivery of twins.

Methods: This retrospective cohort study included all women with twin gestations who choosed an elective cesarean delivery after 28 weeks of gestation at Women's Hospital of Zhejiang University School of $\mathrm{m}$ from September 2014 to April 2019. The Women with an intrauterine fetal demise one or both of twins were excluded.A total of 532 wemen were analysed,they were classified as $\mathrm{PPH}$ group $(n=70)$ and No PPH group $(n=462)$. Estimated blood loss greater than or equal to $1000 \mathrm{ml}$ was defined as Postpartum hemorrhage(PPH).Univariate and multivariate logistic regression analysis were performed to assess the independent risk factors.

Results: Of the 532 twin pregnant women, PPH occurred in $13.2 \%(n=70)$. There were statistically signifcant diferences in preeclampsia $(P=0.005)$, premature rupture of membranes $(P<0.001)$, placental adhesion $(P<0.001)$, placenta previa $(P<0.001)$, hemoglobin $(H b)<100 \mathrm{~g} / L \otimes P=0.003) \otimes$, hematocrit $(H C T)<$

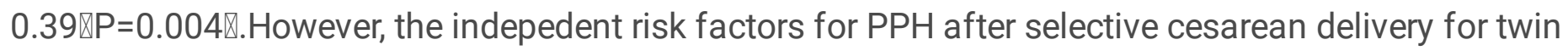
pregnancy were preeclampsia $(\mathrm{OR} 3.538, p<0.05)$, premature rupture of membranes $(\mathrm{OR} 17.9, \mathrm{P}<0.05)$, placental adhesion (OR5.71, $p<0.05)$ and placenta previa (OR10, $p<0.05)$.

Conclusions: preeclampsia, premature rupture of membranes, placental adhesion and placenta previa were risk factors for postpartum hemorrhage after elective cesarean section for twin pregnancy. Risk factor identification and prevention should be a priority.

\section{Background}

In recent years, the incidence of twin pregnancy has increased significantly because of the application and development of assisted reproductive technology(ART)[1]. Twin pregnancy is known to be associated with a higher risk of perinatal morbidity and mortality.Postpartum hemorrhage (PPH) is one of the common complications associated with twin pregnancy, even the leading cause of maternal mortality worldwide[2]. PPH was defined as a cumulative blood loss of greater than or equal to $1,000 \mathrm{~mL}$ or blood loss accompanied by signs or symptoms of hypovolemia within 24 hours after the birth process. Uterine atony is the most common cause of PPH, which accounted for nearly $80 \%$ of the cases [3]. Due to hyperexpansion that damaged the myometrial contractility of uterine, resulting in uterine contraction fatigue, PPH were twofold higher for twin pregnancy [4].

The safest delivery of twin pregnancy has been controversial at many countries.Some investigators have reported risk factors of postpartum hemorrhage after Vaginal delivery of twins[5] .moreover,a report pointed out PPH occurred more often after vaginal delivery than that after elective cesarean delivery for twin pregnancy [6]. However, selective cesarean delivery has been considered as relatively safe for twin pregnancy, because the second fetus in vaginal delivery has a high probability of birth injury and death $[7,8]$. But another report pointed out elective cesarean as a risk factor for transfusion after delivery of 
Twins[9].The rate of cesarean delivery for twin pregnancy has increased significantly in recent years,but few studies have been carried out on factors of PPH in twin pregnancy.The primary goal of this study was to evaluate high risk factors for PPH after elective cesarean section in twin pregnancy by analysis of the clinical data, providing clinical basis for prevention and treatment of $\mathrm{PPH}$.

\section{Materials}

This retrospective study was carried out at Women's Hospital, Zhejiang University School of Medicine from September 2014 to April 2019(Fig. 1). Permission was taken from the Institutional Ethics Committee before starting the study. Exclusion criteria of the study were as follows:1)an intrauterine fetal demise one or both of twins ; 2) gestational weeks less than 28 weeks.

A total of 532 pregnant women were enrolled in the study. The women were divided into two groups according the amount of postpartum hemorrhage:PPH and No PPH. Obstetric records were reviewed区

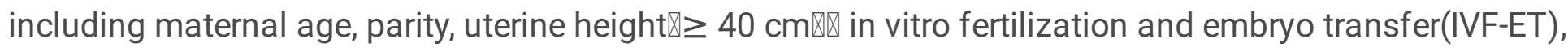
body mass index (BMI $\geq 30$ ), pregnancy diseases (gestational diabetes mellitus, intrahepatic cholestasis during pregnancy, pregnancy hypertension, pre-eclampsia), immune system diseases, chronic hypertension, placental factors (placental adhesion, placenta implantation, placenta previa, placental abruption), scarred uterus $₫$ hysteromyoma, premature rupture of membranes, different chorionic properties, neonatal sex, preoperative laboratory evaluation (hemoglobin, platelet, hematocrit), gestational week ( $\geq 37$ weeks), the sum of the maximum dark zone of amniotic fluid (MVP $\geq 9 \mathrm{~cm}$ ), the sum of twin weight $(\geq 5000 \mathrm{~g})$, anesthesia methods.

All statistical procedures were performed using SPSS 19.0. Qualitative and quantitative variables were described by central and distributive indexes and "Mean $\pm S D$ " was used to explain them.Cases and controls were compared by means of the t-test for continuous variables, and the $\chi 2$ or Fisher's exact test for categorical variables. Differences with $\mathrm{P}<0.05$ were considered significant. Logistic regression analysis was subsequently used to identify the risk factors for postpartum hemorrhage after elective cesarean delivery for twin pregnancy.

\section{Results}

Of the 532 women studied, Oxytocin (20 u) was injected into uterus routinely after delivery of twins delivery during cesarean section. $70 \otimes 13.2 \%$ women underwent postpartum hemorrhage,among which 43 cases were refractory postpartum hemorrhage.In the PPH group, the average amount of bleeding 1314.9 $\pm 419.8 \mathrm{~mL}$, of which 45 patients were treated with blood transfusion. Refractory postpartum hemorrhage refers to the amount of postpartum haemorrhage reached to $1000 \mathrm{ml}$,which failed to stop bleeding with using uterine contraction drugs, therefore required more aggressive treatment such as uterine packing, external compression with uterine sutures, selective devascularization by ligation or embolization of the uterine artery and/or hysterectomy[10]. 


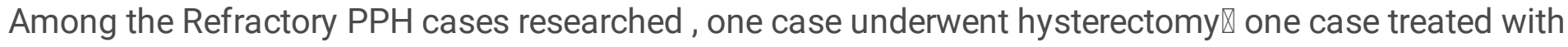
ascending uterine artery ligation and 41 cases used uterine cavity tamponade. In the non-PPH group, the average amount of bleeding was $349.0 \pm 124.6 \mathrm{~mL}$. All of the women had good prognosis without maternal death. There were no significant differences in maternal age, parity, gestational weeks, uterine height, BMI, MVP and twin weight between the two groups $(p<0.05)($ Table 1$)$.

Table1 Analysis of continuous variables by the incidence of postpartum hemorrhage

\begin{tabular}{|llll|}
\hline & No PPH & PPH & P-value \\
\hline Number & 462 & 70 & \\
\hline Maternal age(years) & $31.1+4.0$ & $30.9 \pm 4.2$ & 0.851 \\
\hline Parity & $0.2 \pm 0.4$ & $0.3 \pm 0.5$ & 0.757 \\
\hline Gestational weeks at delivery & $35.9 \pm 1.3$ & $35.7 \pm 1.7$ & 0.732 \\
\hline Twin weight $₫ \mathrm{~g} \rrbracket$ & $5013 \pm 685$ & $5021 \pm 813$ & 0.746 \\
\hline Uterine heigh $\llbracket \mathrm{cm} \rrbracket$ & $39.8 \pm 3.2$ & $39.9 \pm 3.9$ & 0.866 \\
\hline BMI & $28.3 \pm 10.4$ & $27.9 \pm 2.5$ & 0.668 \\
\hline MVP(CM) & $10.2 \pm 2.2$ & $10.8 \pm 3.2$ & 0.314 \\
\hline
\end{tabular}

Data are presented as median (interquartile range) or mean \pm standard deviation. $P P H$ postpartum hemorrhage, BMI body mass index, MVP ,P values in bold indicate signifcant diferences $(P<0.05)$

As show in Table2,the postpartum hemorrhage after elective cesarean delivery for twin pregnancy was associated with preeclampsia $(p=0.005)$, premature rupture of membranes $\varangle P \otimes 0.001 \rrbracket$, placental adhesion

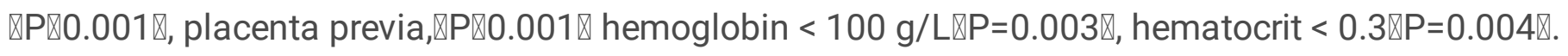

Table2 Analysis of dichotomous variables by the incidence of postpartum hemorrhage 


\begin{tabular}{|c|c|c|c|c|}
\hline & & $\begin{array}{l}\text { No } \\
\text { PPH }\end{array}$ & $\mathrm{PPH}$ & $P$ \\
\hline Nubmer & & 462 & 70 & \\
\hline \multirow[t]{2}{*}{ Maternal age(years) } & $\geq 35$ years & 90 & 12 & 0.67 \\
\hline & $\geq 40$ years & 12 & 3 & 0.68 \\
\hline Parity & $\geq 3$ & 99 & 17 & 0.59 \\
\hline IVF & & 259 & 44 & 0.29 \\
\hline $\mathrm{BMI} \geq 30$ & & 113 & 16 & 0.77 \\
\hline \multirow[t]{4}{*}{ Pregnancy complications } & gestational diabetes mellitus & 71 & 9 & 0.58 \\
\hline & Gestational hypertension & 14 & 6 & 0.1 \\
\hline & Preeclampsia & 25 & 10 & $0.005^{\star}$ \\
\hline & $\begin{array}{l}\text { intrahepatic cholestasis during } \\
\text { pregnancy }\end{array}$ & 28 & 6 & 0.42 \\
\hline Chronic hypertension & & 3 & 0 & 1 \\
\hline Immune systemdiseases & & 3 & 0 & 1 \\
\hline \multirow[t]{4}{*}{ Placental factors } & placenta implantation & 1 & 2 & 0.058 \\
\hline & placental adhesion & 12 & 10 & $0.001 *$ \\
\hline & placental abruption & 1 & 2 & 0.058 \\
\hline & placenta previa & 8 & 10 & $0.001 *$ \\
\hline Uterine height $(>40 \mathrm{~cm})$ & & 254 & 36 & 0.58 \\
\hline Scarred uterus & & 41 & 8 & 0.49 \\
\hline Uterine fibroids & & 14 & 3 & 0.85 \\
\hline Pemature rupture of membranes & & 5 & 6 & $0.001 *$ \\
\hline \multirow{3}{*}{ Chorionicity and Amnionicity } & Dichorionic twins & 370 & 53 & 0.079 \\
\hline & Monochorionic twins & 89 & 17 & \\
\hline & Monoamniotic twins & 3 & 0 & \\
\hline \multirow{2}{*}{$\begin{array}{l}\text { Preoperative laboratory } \\
\text { evaluation }\end{array}$} & hemoglobin $<100 \mathrm{~g} / \mathrm{L}$ & 46 & 16 & $0.003 *$ \\
\hline & hematocrit $<0.3$ & 33 & 13 & $0.004 *$ \\
\hline
\end{tabular}




\begin{tabular}{|c|c|c|c|c|}
\hline & platelet $<100 \times 109 / \mathrm{L}$ & 6 & 3 & 0.069 \\
\hline Fetal weight $(\geq 5000 \mathrm{~g})$ & & 247 & 40 & 0.57 \\
\hline$M V P \rrbracket \geq 9 \mathrm{~cm} \rrbracket$ & & 320 & 49 & 0.90 \\
\hline \multirow[t]{3}{*}{ Neonatal gender } & two boys & 150 & 29 & 0.21 \\
\hline & boy and girl & 149 & 24 & \\
\hline & two girls & 163 & 18 & \\
\hline $\begin{array}{l}\text { Gestational week at delivery ( } \geq \\
37 \text { weeks) }\end{array}$ & & 186 & 30 & 0.08 \\
\hline \multirow[t]{3}{*}{ Anesthesia } & epidural anesthesia & 435 & 56 & 0.07 \\
\hline & $\begin{array}{l}\text { Combined spinal-epidural } \\
\text { anesthesia }\end{array}$ & 26 & 4 & \\
\hline & general anesthesia & 1 & 2 & \\
\hline
\end{tabular}

$\mathrm{PPH}$ postpartum hemorrhage, BMI body mass index, $\mathrm{P}$ values in bold indicate signifcant diferences $\left({ }^{\star} \mathrm{P}<0.05\right)$

Multivariate logstic regression analysis was used to analyze the risk factors of twin pregnancy postpartum hemorrhage after elective cesarean delivery for twin pregnancy .

The results (Table3) showed that preeclampsia (OR3.538, $\mathrm{P}<0.05)$, premature rupture of membranes (OR717.9, $\mathrm{P}<0.05)$, placental adhesion (OR5.71, $\mathrm{P}<0.05)$ and placenta previa $(\mathrm{OR} 10, \mathrm{P}<0.05)$ were the independent risk factors for postpartum hemorrhage after elective cesarean delivery for twin pregnancy.

Table3 Results of multivariable logistic regression model to predict Risk factors for after elective cesarean delivery for twin pregnancy 


\begin{tabular}{|c|c|c|c|c|}
\hline Characteristic & No PPH & $\mathrm{PH}$ & OR & $\mathrm{P}$ \\
\hline Preeclampsia & 25 & 10 & 3.53 & 0.011 \\
\hline Pemature rupture of Membranes & 5 & 3 & 17.9 & $\otimes 0.001$ \\
\hline Placental adhesion & 12 & 10 & 5.71 & 0.001 \\
\hline Placenta previa & 8 & 10 & 10.0 & $\bowtie 0.001$ \\
\hline Hemoglobin $<100 \mathrm{~g} / \mathrm{L}$ & 46 & 16 & 0.971 & 0.388 \\
\hline Hematocrit $<0.3$ & 33 & 13 & 0.884 & 0.821 \\
\hline
\end{tabular}

\section{Discussion}

The incidence rate and mortality of twin pregnancy in perinatal period are higher than that of single pregnancy. Our study showed a similar PPH rate (13.2) of elective cesarean section in tiwn pregnancy with that of some studies (14\%) [7]. However, it is also reported this rate after elective cesarean section in tiwn pregnancy could be as low as $5.1 \%$, meanwhile this number after vaginal delivery in twin pregnancy was $17.7 \%$ [9]. This may due to the different physical conditions of pregnant women in these studies. Twin pregnancy also is a high risk factor for PPH[11]. Some scholars had compared different high risk factors of PPH between singleton pregnancy and twin pregnancy, it was premature delivery, placenta previa and placenta abruption for the former, while hypertension and gestational weeks $(\geq 41)$ for the latter [12].

Preeclampsia was shown to be an independent risk factor for PPH after elective cesarean section in twin pregnancy in the present study, the reason has not yet been clearly understood. As suggested by Minakami's report [13], the reason of preeclampsia inducing PPH may be caused by gradual decrease of the activity of antithrombin III or platelet during the second trimester of twin pregnancy. However, only one case of PPH complicated by preeclampsia was accompanied by thrombocytopenia in our study, but the activity of antithrombin III had neither been tested. Preeclampsia is a common complication of twin pregnancy and a high risk factor of elective cesarean section in twin pregnancy. It also suggested that women with preeclampsia in twin pregnancy should be early diagnosed and managed to prevent or reduce the occurrence of $\mathrm{PPH}$, and improve the prognosis of pregnant women. Moreover, our study

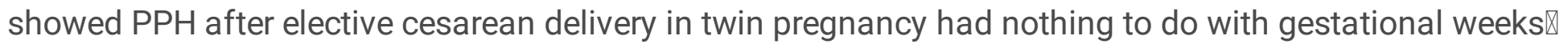
which is different from the present study. The reason of this difference might be that the maximum termination time of twin pregnancy was only 38 weeks.

Because of the high pressure of uterine cavity in twin pregnancy, the probability of premature rupture of membranes is $7-8 \%[14-15]$.After premature rupture of membranes, the role of membranes as a barrier 
disappears, so pathogens are more likely to invade the uterine cavity, leading to chorioamnionitis. Chorioamnionitis is a high risk factor for postpartum hemorrhage [16]. Premature rupture of membranes and chorioamnionitis are causal factors, which may also be a high risk factor for postpartum hemorrhage. The results of retrospective analysis suggested premature rupture of membranes was an independent risk factor for postpartum hemorrhage after selective cesarean section, which is consistent with the previou conclusions. But in our study, 11 cases of premature rupture of membranes had no clinical symptoms of chorioamnionitis. Only 3 cases of premature rupture of membranes supported chorioamnionitis pathologically. Therefore, the cause of postpartum hemorrhage caused by premature rupture of membranes in this study could not be completely determined as chorioamnionitis.

The retrospective analysis showed that placenta previa and placental adhesion were independent risk factors for postpartum hemorrhage after elective cesarean delivery for twin pregnancy. Because placental villi invade the superficial myometrium of the uterus, the adherent placenta can not be detached when removing placenta, resulting in poor uterine contraction and bleeding of the detached placenta sinus. Because of the low placenta position, large dissection surface, poor contraction of the lower uterine segment and open blood sinus, placenta previa bleeds .In the PPH group囚placenta previa and placental adhesion have accounted for $14.2 \%$, of which $27.9 \%$ were intractable postpartum hemorrhage. Therefore, for twin pregnancy with placenta adhesions and placenta previa, if the bleeding is more during surgery and the drug treatment is ineffective, We should as soon as possible take other forms of treatment such as uterine packing, external compression with uterine sutures and selective devascularization by ligation to avoid blood transfusion, disseminated intravascular coagulation(DIC), or even hysterectomy. The retrospective analysis showed that placenta implantation and placental abruption were not independent risk factors for postpartum hemorrhage after elective cesarean delivery for twin pregnancy. The results may be related to fewer cases .

- There are also reports about risk factors for postpartum hemorrhage after elective cesarean delivery for twin pregnancy including advanced age, IVF, general anesthesia and so on[9.17.18]. However, in the study $\llbracket$ elderly pregnant women is account for 19\%『Patients with PPH and NPPH in vitro infants accounted for halfhalf ; caesarean section more use epidural anesthesia, general anesthesia less at our hospital.Considering the possible reasons of sample size, the results of this retrospective analysis are different from other studies, and need further research.

Twin pregnancy and selective cesarean section are high risk factors for postpartum hemorrhage, so the rate of postpartum hemorrhage is very high for pregnant women with selective cesarean section in twin pregnancy. The retrospective analysis showed that preeclampsia, placental adhesion, placenta previa and premature rupture of membranes were high risk factors for postpartum hemorrhage after elective cesarean delivery for twin pregnancy. Clinicians should fully assess $₫$ the multiple interventions, various oxytocin drugs, preventive intrauterine tamponade, or local uterine suture for high risk factors for twin pregnancies, and actively prevent postpartum hemorrhage. Reduce the incidence and mortality of postpartum hemorrhage in twins. 


\section{Conclusions}

Clinicians should fully evaluate Risk factors for postpartum hemorrhage after elective cesarean delivery for twin pregnancy, multiple interventions can be considered before the operation,such as a variety of uterine contractions, preventive uterine tamponade, or local uterine sutures $₫$ to actively prevent postpartum hemorrhage and reduce incidence and mortality of postpartum hemorrhage for twin pregnancy.

At present, many scholars are trying to establish predictive models of PPH for for twin pregnancy. we also hope to expand the number of samples future, develop and verify models for predicting postpartum hemorrhage in twin pregnancy .

\section{Abbreviations}

PPH:Postpartum hemorrhage;HB:hemoglobin;HCT:hematocrit;ART:assisted reproductive technology;IVFET:in vitro fertilization and embryo transfer;BMl:body mass index ;MVP:the sum of the maximum dark zone of amniotic fluid.

\section{Declarations}

Ethical approval and consent to participate: This study was supervised and approved by the ethics committee of The Women's Hospital, School of Medicine, Zhejiang University, China.For this type of study formal consent is not required.

Consent for publication: We have obtained consent to publish from each patient.

Availability of data and materials: We have had full control of all primary data and we agree to allow the Journal and readers to review our data if requested.

Competing interest :The authors declare that they have no conflict of interest.

Funding:This study was not supported .

Author Contribution:

Xiaojie Wan : Data Collection, Data analysis and Manuscript writing

Wei Zhao:Data Collection

Li Zhao:Data analysis

Hong Wen: Conception and design of the study

\section{Author details}


Women's Hospital,School of Medicine, Zhejiang University , 1 Xueshi Road,Hangzhou, Zhejiang Province 310006, China

corresponding authorखHong Wen, 5199002@zju.edu.cn

First author:Xiaojie Wan,5518006@zju.edu.cn

\section{References}

1. Slattery MM, Morrison JJ. Preterm delivery. Lancet 2002;360:1489-1497.

2. Committee on Practice B-O. Practice bulletin No. 183:postpartum hemorrhage. Obstet Gynecol. 2017;130(4):e168-e186.

3. Bateman BT, Berman MF, Riley LE, et al. The epidemiology of postpartum hemorrhage in a large, nation wide sample of deliveries. Anesth Analg. 2010;110:1368-1373.

4. Santana DS, Cecatti JG, Surita FG, et al. Twin pregnancy and severe maternal outcomes: the world health organization multicountry survey on maternal and newborn health. Obstet Gynecol. 2016;127:631-641.

5. Shunji Suzuki ,Yoshie Hiraizumi ,Hidehiko Miyake.Risk factors for postpartum hemorrhage requiring transfusion in cesarean deliveries for Japanese twins: comparison with those for singletons.Arch Gyn.2012;286:1363-1367

6. Dana Sadeh-Mestechkin, Yair Daykan, Mor Bustan囚et al.Trial of vaginal delivery for twins - is it safe? A single center experience.The Journal of Maternal-Fetal \& Neonatal Medicine.2017;31:15, 1967-1971.

7. Yang Q, Wen SW, Chen Y, et al. Neonatal mortality and morbidity in vertex-vertex second twins according to mode of delivery and birth weight. J Perinatol.2006;26:3-10.

8. Yang Q, Wen SW, Chen Y, et al. Neonatal death and morbidity in vertex-non vertex second twins 1970 D. SADEH-MESTECHKIN ET AL. according to mode of delivery and birth weight. Am J Obstet Gynecol. 2005;192:840-847.

9. Shunji Suzuki, Yusuke Inde, Miwa Igarash囚et al.Elective Cesarean as a Risk Factor for Transfusion after Delivery of Twins.J Nippon Med Sch 2008; 75: 247-249).

10. Pardo Ml, Trujillo M V, Campos S, ct al. Successful uterine artery embolization for intractable postpartum hemorrhage using a novel embolic agent: ONYX J . J Gynecol Surgery. 2013. 29 ( 1 )凹 309-311.

11. Souza JP, Gülmezoglu AM, Vogel J, Carroli G, Lumbiganon P,Qureshi Z, et al. Moving beyond essential interventions for reduction of maternal mortality (the WHO Multicountry Survey on Maternal and Newborn Health): a cross-sectional study.Lancet 2013;381:1747-55.

12. Shunji Suzuki ,Yoshie Hiraizumi ,Hidehiko Miyake.Risk factors for postpartum hemorrhage requiring transfusion in cesarean deliveries for Japanese twins: comparison with those for singletons.Arch Gynecol Obstet (2012) 286:1363-1367. 
13. Minakami H, Watanabe T, Izumi A, Matsubara S, Koike T,Sayama M, Moriyama I, Sato I (1999) Association of a decrease in antithrombin Illactivity with a peripheral elevation in asparatate aminotransferase in women with twin pregnancies: relevance to the HELLP syndrome. $J$ Hepatol 30:603-611.

14. Mercer BM. Preterm premature rupture of the membranes. Obstetrics and gynecology 2003;101:17893.

15. Melamed N, Ben-Haroush A, Pardo J, et al. Expectant management of preterm premature rupture of membranes: is it all about gestational age? American journal of obstetrics and gynecology 2011;204:48 e1-8.

16. Magann EF, Evans S, Hutchinson M, Collins R,Lanneau G, Morrison JC. Postpartum hemorrhage after cesarean delivery: an analysis of risk factors. South Med J.2005;98:681

17. Dana Sadeh-Mestechkin, Yair Daykan, Mor Bustan, Ofer Markovitch, Gil Shechter-Maor \& Tal BironShental.Trial of vaginal delivery for twins - is it safe? A single center experience.The Journal of Maternal-Fetal \& Neonatal Medicine, 31:15, 1967-1971.

18. Caixia Zhu, Malie Wang, Gang Niu囚et al.Obstetric outcomes of twin pregnancies at advanced maternal age: A retrospective study.Taiwanese Journal of Obstetrics \& Gynecology 2018;57:64-67.

\section{Figures}




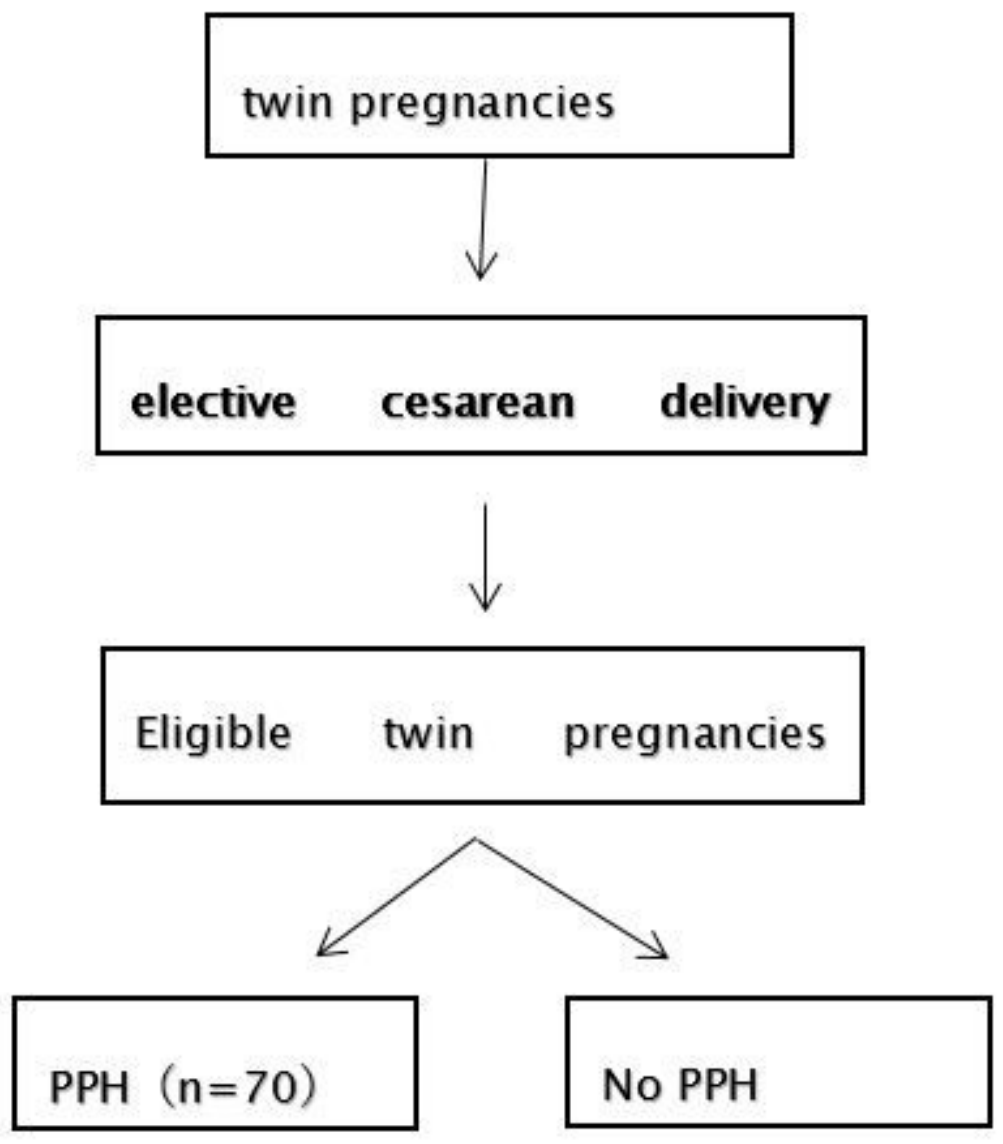

Figure 1

Flow sheet for inclusion criteria 